\title{
Viral hepatitis status does not affect survival in patients with hepatocellular carcinoma
}

\author{
Eyas Alkhalili, Alissa Greenbaum, Li Luo, Rodrigo Rodriguez, Katharine Caldwell, Oscar Munoz Estrada, \\ Jacqueline O'Neill, Itzhak Nir, Katherine T. Morris
}

University of New Mexico, New Mexico, USA

\section{Abstract}

\begin{abstract}
Background There have been few studies on the impact of viral etiology on the prognosis in patients with hepatocellular carcinoma (HCC). The aim of this study was to evaluate the clinical characteristics and survival of patients with viral hepatitis-associated HCC (V-HCC), compared to patients with HCC of non-hepatitis B, non-hepatitis C (NBNC-HCC) etiology.
\end{abstract}

Methods We performed a retrospective analysis of all patients with HCC treated at our comprehensive cancer center from 2000 through 2014. Patients were divided into two groups according to their viral hepatitis status. Presentation patterns, treatments, and survival data were analyzed.

Results We evaluated 366 patients: 233 patients (63.7\%) had V-HCC while 133 (36.3\%) patients had NBNC-HCC. V-HCC patients were younger $(\mathrm{P}<0.0001)$ and more likely to be male $(\mathrm{P}=0.001)$. Decompensated cirrhosis was more prevalent in $\mathrm{V}$-HCC patients $(\mathrm{P}=0.01)$. There was no difference in the resectability rate or disease stage. In patients with resectable disease, those with V-HCC were less likely to undergo hepatectomy $(23.7 \%$ vs. $38 \%$; $\mathrm{P}=0.04)$ for more advanced liver disease. The estimated median survival for V-HCC was 13 months compared to 16 months in NBNCHCC patients $(\mathrm{P}=0.57)$. On multivariate analysis, disease stage $(\mathrm{P}<0.0001)$ and Child-Pugh class $(\mathrm{P}<0.0001)$ were independent factors affecting survival, but viral status was not $(\mathrm{P}=0.75)$.

Conclusion Despite presenting with more advanced cirrhosis and being less likely to undergo surgery, V-HCC patients had similar survival to patients with NBNC-HCC.

Keywords Hepatocellular carcinoma, survival, viral hepatitis

Ann Gastroenterol 2017; 30 (1): 1-6

\section{Introduction}

Hepatocellular carcinoma (HCC) is the fifth most common cancer worldwide and its incidence in the United States is increasing [1,2]. HCC usually arises in the setting of chronic liver disease, with $70-90 \%$ of patients having chronic liver disease and cirrhosis [3,4]. Worldwide, 75\% of HCC cases are associated with chronic viral hepatitis. While hepatitis B is implicated in about half of cases worldwide, $40-60 \%$ of HCC cases in the United States and Europe are associated with hepatitis $\mathrm{C}$ virus,

Department of Surgery, School of Medicine, University of New Mexico, New Mexico, USA

Conflict of Interest: None

Correspondence to: Eyas Alkhalili, MD, Office 1, UNM HSC Department of Surgery, MSC 10 5610, 1 UNM, Albuquerque, New Mexico 87131, USA, e-mail: EAlkhalili@salud.unm.edu

Received 16 August 2016; accepted 29 September 2016; published online 20 October 2016

DOI: https://doi.org/10.20524/aog.2016.0097 making it the most common offending agent $[1,5]$. There have been few studies of the impact of viral etiology on the prognosis in patients with HCC. While some studies have shown that the cause of cirrhosis did not affect the overall prognosis [6], others, particularly from Asia, have shown a significant association between HCC prognosis and the etiology of liver disease $[7,8]$.

Given the lack of consensus on the significance of viral hepatitis in the overall prognosis in HCC, and the paucity of studies examining these differences, we aimed to investigate the association between presentation patterns, treatments, and prognosis in HCC patients with cirrhosis and chronic liver disease etiology.

\section{Patients and methods}

A retrospective chart review of all patients with HCC treated at our institution from 2000 through 2014 was performed after Institutional Review Board approval had been obtained. The Comprehensive Cancer Center at the University of New Mexico is the only designated National Cancer Institute 
tertiary care center in the state of New Mexico, making it the major referral center for patients with HCC.

HCC patients were divided into two groups: those with a history of chronic viral hepatitis $\mathrm{B}$ or $\mathrm{C}$ and viral-associated HCC (V-HCC), and those with HCC of non-hepatitis B, nonhepatitis $\mathrm{C}$ (NBNC-HCC) etiology.

We defined technical resectability by the absence of extrahepatic disease, adequate future liver remnant, and no tumor involvement of the inferior vena cava, main portal vein, or main hepatic artery. The proportion of patients who were offered surgery (among those with technically resectable disease), chemotherapy and/or interventional radiological therapy, such as radiofrequency ablation or transarterial chemoembolization, were recorded. Liver transplantation is not available in the state of New Mexico. While appropriate candidates are referred to another centers, these patients were not included in our analysis.

\section{Statistical analysis}

Patients' demographics, etiology of cirrhosis, Child-Pugh classification, AJCC $7^{\text {th }}$ edition disease stage at diagnosis, and the treatments offered and received were compared using the Student's $t$-test and ANOVA for continuous variables, and chisquare tests for categorical variables. All statistical tests were two-tailed and $\mathrm{P}<0.05$ was considered statistically significant.

The overall survival was compared by Kaplan-Meier curves using SPSS software version 14.0 (SPSS, Chicago, IL, USA). Cox regression models were generated to calculate the hazard ratio (HR) and 95\% confidence intervals (CI). Multivariate analysis was performed using the SAS software (SAS Institute, Cary, NC, USA).

\section{Results}

We evaluated 366 patients (Table 1): 233 patients (63.7\%) had V-HCC while 133 (36.3\%) patients had NBNC-HCC. In the V-HCC group, 207 patients (88.8\%) had hepatitis C, $16(6.9 \%)$ had hepatitis B, and 10 (4.3\%) had both. The mean age at the time of diagnosis in the V-HCC groups was significantly lower than that of the NBNC-HCC group (56 \pm 8.3 years vs. $65 \pm 11.1$ years; $\mathrm{P}<0.0001)$. Male predominance

Table 1 Patient demographics, clinical presentation and treatments data

\begin{tabular}{|c|c|c|c|}
\hline & $\begin{array}{c}\text { V-HCC }(\mathrm{n}=233) \\
(\%)\end{array}$ & $\begin{array}{c}\text { NBNC-HCC }(\mathrm{n}=133) \\
(\%)\end{array}$ & $\mathrm{P}$-value \\
\hline Sex & & & 0.001 \\
\hline Male & $198(84.9)$ & $97(70.6)$ & \\
\hline Female & $35(15.1)$ & $36(29.4)$ & \\
\hline Age in years & & & $<0.0001$ \\
\hline Mean \pm SD & $56 \pm 8.3$ & $65 \pm 11.1$ & \\
\hline Ethnicity & & & 0.003 \\
\hline Hispanic & $119(51)$ & $64(48.10)$ & \\
\hline Native American & $14(6)$ & $23(17.30)$ & \\
\hline Non-Hispanic White & $70(30)$ & $36(27)$ & \\
\hline Other & $30(12.90)$ & $10(7.60)$ & \\
\hline Child-Pugh class & & & 0.01 \\
\hline None & $6(2.60)$ & $22(16.50)$ & \\
\hline A & $90(38.60)$ & $50(37.60)$ & \\
\hline B & $79(34)$ & $42(31.60)$ & \\
\hline $\mathrm{C}$ & $58(24.80)$ & $19(14.30)$ & \\
\hline Stage at presentation & & & 0.23 \\
\hline I & $57(24.50)$ & $40(30)$ & \\
\hline II & $65(27.90)$ & $25(18.80)$ & \\
\hline III & $61(26.10)$ & $35(26.30)$ & \\
\hline IV & $50(21.50)$ & $33(24.90)$ & \\
\hline Technically resectable & $131(56.20)$ & $71(53.30)$ & 0.59 \\
\hline Hepatectomy performed in resectable disease & $31 / 131(23.7)$ & $27 / 71(38)$ & 0.04 \\
\hline Chemotherapy & $52(22.30)$ & $33(24.80)$ & 0.58 \\
\hline IR & $85(36.50)$ & $52(39)$ & 0.61 \\
\hline
\end{tabular}

V-HCC, viral-associated hepatocellular carcinoma; NBNC-HCC, non-hepatitis B, non-hepatitis C HCC; IR, interventional radiology; SD, standard deviation 
was more pronounced in the V-HCC group, with 198 male patients $(84.9 \%)$ vs. 94 males $(70.6 \%)$ in the NBNC-HCC group $(\mathrm{P}=0.001)$. There was a statistically significant difference in the ethnic distribution between the two groups, with a higher proportion of Native American patients in the NBNCHCC group $(\mathrm{P}=0.003)$.

Patients with V-HCC were more likely to present with decompensated cirrhosis compared to those with NBNC-HCC. While only $2.6 \%$ of V-HCC patients did not have a diagnosis of cirrhosis, as many as $16.5 \%$ of NBNC-HCC patients had no cirrhosis. Child-Pugh class was also higher in the V-HCC group, with $24.8 \%$ of patients having class $\mathrm{C}$ cirrhosis compared to $14.3 \%$ in the NBNC-HCC group ( $\mathrm{P}=0.01$ for all of the above).

There was no difference in the disease stage at presentation between the two groups $(\mathrm{P}=0.23)$. The technical resectability rate was similar between the two groups $(56.2 \%$ in V-HCC vs. $53.3 \%$ in NBNC-HCC; $\mathrm{P}=0.59$ ). However, because of the higher incidence of decompensated cirrhosis in the V-HCC group, these patients were less likely to be offered and eventually undergo hepatectomy (23.7\% vs. $38 \%$; $\mathrm{P}=0.04)$.

The proportion of patients receiving chemotherapy was similar in both groups $(22.3 \%$ vs. $24.8 \% ; \mathrm{P}=0.58)$. There was no difference in the rate of receiving transarterial chemoembolization and/or radiofrequency ablation therapies (36.5\% vs. $39 \%$; $\mathrm{P}=0.61$ ).

The estimated median overall survival in the V-HCC group was 13 months compared to 16 months in NBNC-HCC, but this was not statistically significant $(\mathrm{P}=0.57)$ (Fig. 1). No differences were found in stage-specific median survivals for stage I (36 months in V-HCC vs. 29 months NBNC-HCC), stage II (13 vs. 14 months), stage III (6 vs. 7 months), and stage IV (5 vs. 6 months) (Fig. 2). Multivariate analysis showed that, while the presence of viral hepatitis was not an independent factor affecting overall survival $(\mathrm{P}=0.75)$, ethnicity $(\mathrm{P}=0.0008)$, disease stage $(\mathrm{P}<0.0001)$, and Child-Pugh class $(\mathrm{P}<0.0001)$ were significant elements in determining survival (Table 2).

\section{Discussion}

Our study demonstrated differences in the presentation patterns between V-HCC and NBNC-HCC, with viral patients presenting at a younger age, with more advanced cirrhosis, and with a lower probability of undergoing surgical resection than their NBNC-HCC counterparts. Despite these differences, no difference in the disease prognosis was found.

Male predominance was more pronounced in the V-HCC group in our cohort but sex was not an independent factor affecting survival. Even after adjusting for confounding variables such as increased incidence of viral hepatitis and alcoholic cirrhosis, men have been shown to be at greater risk of HCC than women [1]. Tangkijvanich et al showed that males tend to present with advanced disease and have worse survival than females (median survival 4 vs. 14 months, $\mathrm{P}=0.004$ ) [9], while Farinati et al demonstrated that females had a better

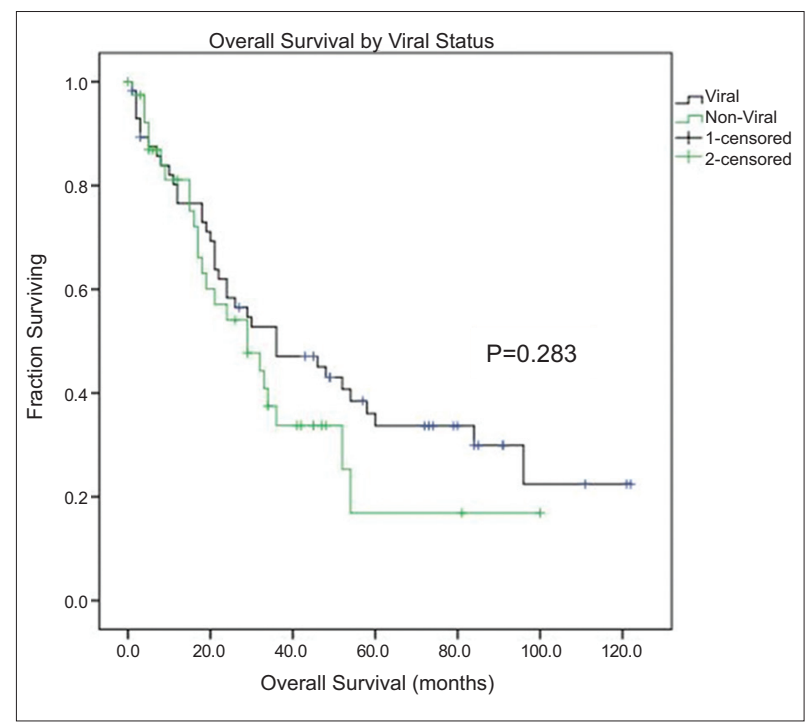

Figure 1 Kaplan-Meier overall survival curves by viral status

Table 2 Multivariate analysis of independent factors affecting survival

\begin{tabular}{|c|c|c|c|}
\hline Effect & $\mathrm{HR}$ & $95 \%$ CI & P-value \\
\hline HCC etiology & & & 0.75 \\
\hline Viral & Ref & - & \\
\hline NBNC & 0.95 & $0.72-1.27$ & \\
\hline Ethnicity & & & 0.0008 \\
\hline $\begin{array}{l}\text { Non-Hispanic } \\
\text { White }\end{array}$ & Ref & - & \\
\hline Asian & 0.46 & $0.22-0.94$ & \\
\hline Black & 0.60 & $0.24-1.52$ & \\
\hline Hispanic & 1.27 & $0.96-1.67$ & \\
\hline Native American & 0.55 & $0.34-0.90$ & \\
\hline Age & 1 & $0.68-1.02$ & 0.19 \\
\hline Sex & & & 0.99 \\
\hline Female & Ref & - & \\
\hline Male & 1 & $0.73-1.37$ & \\
\hline Child-Pugh class & & & $<0.0001$ \\
\hline None & Ref & - & \\
\hline A & 0.97 & $0.59-1.6$ & \\
\hline B & 1.54 & $0.93-2.55$ & \\
\hline $\mathrm{C}$ & 2.5 & $1.44-4.30$ & \\
\hline Stage & & & $<0.0001$ \\
\hline I & Ref & - & \\
\hline II & 1.6 & $1.14-2.35$ & \\
\hline III & 2.4 & $1.74-3.49$ & \\
\hline IV & 3.1 & $2.20-4.55$ & \\
\hline
\end{tabular}

HR, hazard ratio; CI, confidence interval; HCC hepatocellular carcinoma; $N B N C$, non-hepatitis $B$, non-hepatitis $C$ 


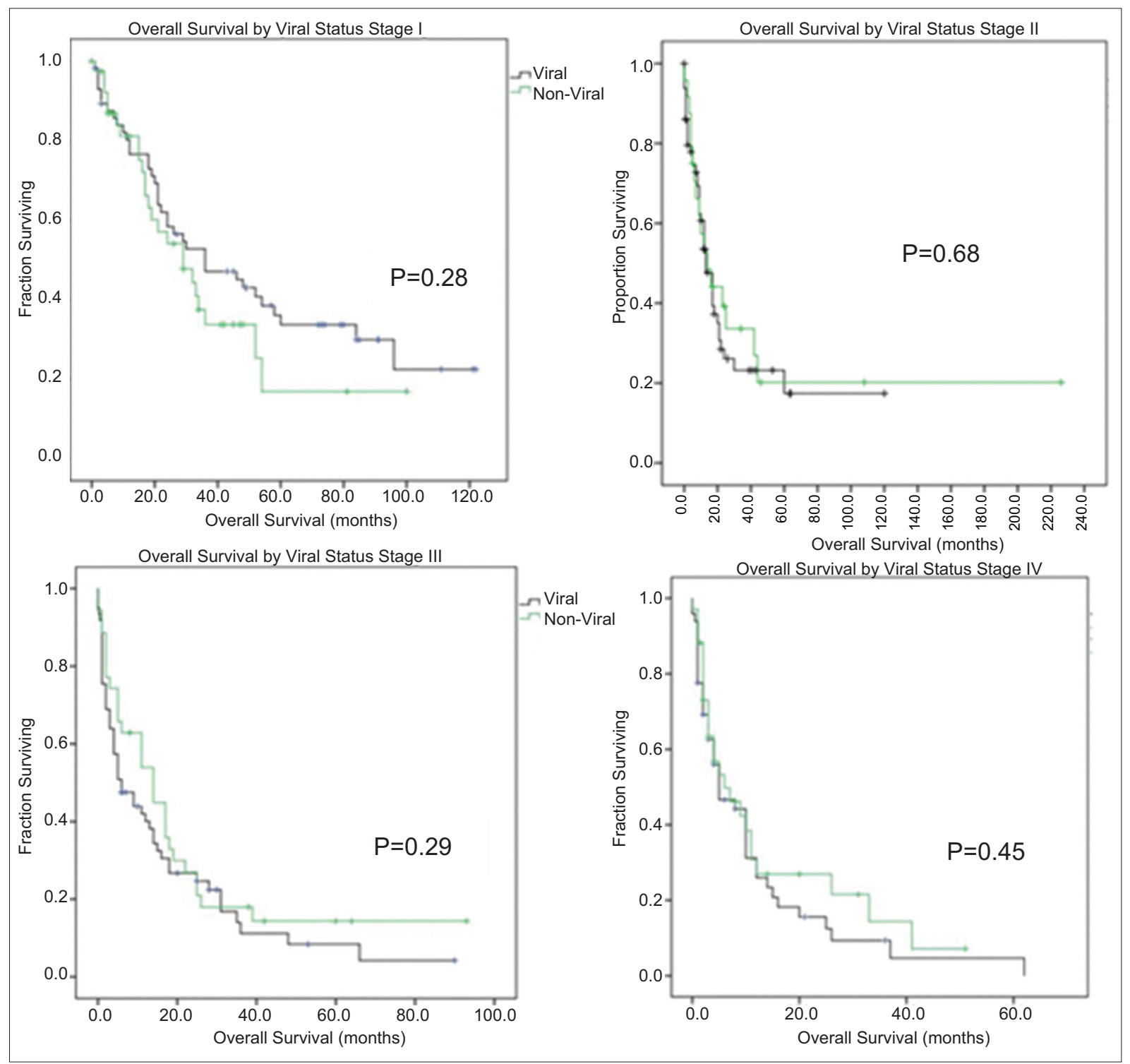

Figure 2 Stage-specific Kaplan-Meier survival curves by viral status

prognosis, possibly because they presented in earlier stages and showed better compliance with cancer surveillance [10]. Several other analyses, however, have shown that sex was not an independent variable for survival [11-14]. Targeting hormonal receptors as a treatment method of HCC has proven ineffective $[15,16]$.

The younger age of patients in the V-HCC group, as well as the higher incidence of decompensated cirrhosis compared to the NBNC-HCC group, suggest a faster progression of liver disease with viral hepatitis. The majority of studies comparing the prognosis of HCC according to viral status come from Japan, where hepatitis $C$ is the predominant causative agent $[7,17,18]$. Akahoshi et al showed that the cause of cirrhosis was an independent factor in determining survival. In their study, while NBNC-HCC patients presented more commonly with advanced disease, stage-stratified comparisons found that NBNC-HCC patients fared better than those with V-HCC [7]. Toyoda et al studied 1152 patients with HCC, 10\% of whom were viralmarker negative. In that study, NBNC-HCC patients were less likely to be under surveillance for HCC, presented with advanced stage disease, and had a worse prognosis. Our cohort of patients had a larger proportion of NBNC-HCC related to alcohol or nonalcoholic steatohepatitis. Regardless of the differences in presentation and treatment between the two groups, the overall prognosis was not different. Presenting at a younger age, or a different tumor biology may explain why viral patients had a similar prognosis to their non-viral counterparts. A recent metaanalysis showed that adjuvant interferon therapy after curative treatment for viral-associated HCC improved survival and decreased the recurrence rate [19]. This is in contrast to patients with NBNC-HCC who receive no therapeutic agents targeting the underlying cause of liver disease. 
Disease stage and Child-Pugh score were strong predictors of overall survival, consistent with previous literature. The majority of staging systems for HCC, including the Barcelona Clinic Liver Cancer, Cancer of Liver Italian Program, and Japan Integrated Scoring, incorporate the stage of the underlying cirrhosis in predicting survival [20-22]. We used the TNM staging system and found similar stage distribution between the two groups. Although the other staging systems account for cirrhosis, while the TNM does not, the TNM staging system was an independent factor in prognosis and it is the preferred staging system at our institution.

Despite a higher incidence of decompensated cirrhosis in V-HCC patients, we found their prognosis was not different from NBNC-HCC patients. The Italian Liver Cancer Group studied 742 patients with HCC detected on annual surveillance and found no difference in survival related to the etiology of the disease, which is in accordance with our results [6]. Another study by the same group demonstrated a difference in survival in advanced HCC between different types of hepatitis, as patients with hepatitis B had better survival than those associated with hepatitis C (hazard ratio [HR] 1.5, 95\% confidence interval [CI] 1-2.29, $\mathrm{P}=0.048$ ) [23]; however, this has not been consistent in the literature. Samonakis et al found contradictory results, with hepatitis B being associated with twice the risk of death of patients with hepatitis $C$ [24]. The majority of our V-HCC patients had hepatitis C, which makes this patient group overrepresented when compared to hepatitis $\mathrm{B}$, and the results might be more reflective of these patients' prognosis.

Our study is limited by its single-center setting and retrospective nature. A difference in disease presentation and prognosis may exist in populations other than the ethnic groups represented in our state. We confirmed there was no

\section{Summary Box}

\section{What is already known:}

- Hepatocellular carcinoma (HCC) arises in the setting of chronic liver disease and cirrhosis

- Viral hepatitis is the most common cause of liver disease and HCC

- HCC has multiple staging systems and has a complex multimodality treatment algorithm

\section{What the new findings are:}

- Viral hepatitis is associated with earlier age at diagnosis of HCC

- Patients with HCC associated with viral hepatitis have more advanced cirrhosis and are less likely to undergo surgery

- Viral hepatitis is not an independent prognostic factor in HCC difference in presentation stage, but we did not account for differences in surveillance patterns, which may have varied between the two groups.

In conclusion, in our series, patients with V-HCC were more likely to be men and to present at a younger age. Furthermore, V-HCC patients had a higher rate of decompensated cirrhosis, which made them less likely to undergo surgical resection. Despite these discrepancies, V-HCC patients had a similar prognosis to their NBNCHCC counterparts. The effect of antiviral therapy may be an important adjuvant treatment, without a counterpart available for patients with NBNC-HCC.

\section{References}

1. El-Serag HB. Epidemiology of viral hepatitis and hepatocellular carcinoma. Gastroenterology 2012;142:1264-1273.e1

2. El-Serag HB, Davila JA, Petersen NJ, McGlynn KA. The continuing increase in the incidence of hepatocellular carcinoma in the United States: an update. Ann Intern Med 2003;139:817-823.

3. El-Serag HB, Rudolph KL. Hepatocellular carcinoma: epidemiology and molecular carcinogenesis. Gastroenterology 2007;132:2557-2576.

4. Poon D, Anderson BO, Chen LT, et al; Asian Oncology Summit. Management of hepatocellular carcinoma in Asia: consensus statement from the Asian Oncology Summit 2009. Lancet Oncol 2009;10:1111-1118.

5. Gurtsevitch VE. Human oncogenic viruses: hepatitis B and hepatitis C viruses and their role in hepatocarcinogenesis. Biochemistry (Mosc) 2008;73:504-513.

6. Trevisani F, Magini G, Santi V, et al; Italian Liver Cancer (ITA. LI.CA) Group. Impact of etiology of cirrhosis on the survival of patients diagnosed with hepatocellular carcinoma during surveillance. Am J Gastroenterol 2007;102:1022-1031.

7. Akahoshi $\mathrm{H}$, Taura $\mathrm{N}$, Ichikawa $\mathrm{T}$, et al. Differences in prognostic factors according to viral status in patients with hepatocellular carcinoma. Oncol Rep 2010;23:1317-1323.

8. Kondo K, Chijiiwa K, Funagayama M, Kai M, Otani K, Ohuchida J. Differences in long-term outcome and prognostic factors according to viral status in patients with hepatocellular carcinoma treated by surgery. J Gastrointest Surg 2008;12:468-476.

9. Tangkijvanich P, Mahachai V, Suwangool P, Poovorawan Y. Gender difference in clinicopathologic features and survival of patients with hepatocellular carcinoma. World J Gastroenterol 2004;10:1547-1550.

10. Farinati F, Sergio A, Giacomin A, et al; Italian Liver Cancer group. Is female sex a significant favorable prognostic factor in hepatocellular carcinoma? Eur J Gastroenterol Hepatol 2009;21:1212-1218.

11. El-Serag HB. Hepatocellular carcinoma: an epidemiologic view. J Clin Gastroenterol 2002;35(5 Suppl 2):S72-S78.

12. Qin LX, Tang ZY. The prognostic molecular markers in hepatocellular carcinoma. World J Gastroenterol 2002;8:385-392.

13. Shimoda M, Ghobrial RM, Carmody IC, et al. Predictors of survival after liver transplantation for hepatocellular carcinoma associated with Hepatitis C. Liver Transpl 2004;10:1478-1486.

14. Sherman M. Hepatocellular carcinoma: epidemiology, risk factors, and screening. Semin Liver Dis 2005;25:143-154.

15. Di Maio M, De Maio E, Morabito A, et al. Hormonal treatment of human hepatocellular carcinoma. Ann N Y Acad Sci 2006;1089:252-261.

16. Di Maio M, Daniele B, Pignata S, et al. Is human hepatocellular 
carcinoma a hormone-responsive tumor? World J Gastroenterol 2008;14:1682-1689.

17. Dohmen K, Shigematsu H, Irie K, Ishibashi H. Comparison of the clinical characteristics among hepatocellular carcinoma of hepatitis $\mathrm{B}$, hepatitis $\mathrm{C}$ and non-B non-C patients. Hepatogastroenterology 2003;50:2022-2027.

18. Toyoda H, Kumada T, Kiriyama S, et al. Characteristics and prognosis of patients in Japan with viral marker-negative hepatocellular carcinoma. J Gastroenterol Hepatol 2008;23:459-466.

19. Xu J, Li J, Chen J, Liu ZJ. Effect of adjuvant interferon therapy on hepatitis $\mathrm{b} / \mathrm{c}$ virus-related hepatocellular carcinoma after curative therapy - meta-analysis. Adv Clin Exp Med 2015;24:331-340.

20. Guglielmi A, Ruzzenente A, Pachera S, et al. Comparison of seven staging systems in cirrhotic patients with hepatocellular carcinoma in a cohort of patients who underwent radiofrequency ablation with complete response. Am J Gastroenterol 2008;103:597-604.

21. Hsu CY, Hsia CY, Huang YH, et al. Selecting an optimal staging system for hepatocellular carcinoma: comparison of 5 currently used prognostic models. Cancer 2010;116:3006-3014.

22. Liu PH, Hsu CY, Hsia CY, et al. Prognosis of hepatocellular carcinoma: Assessment of eleven staging systems. J Hepatol 2016;64:601-608.

23. Cantarini MC, Trevisani F, Morselli-Labate AM, et al; Italian Liver Cancer (ITA.LI.CA) group. Effect of the etiology of viral cirrhosis on the survival of patients with hepatocellular carcinoma. Am J Gastroenterol 2006;101:91-98.

24. Samonakis DN, Koulentaki M, Coucoutsi C, et al. Clinical outcomes of compensated and decompensated cirrhosis: A long term study. World J Hepatol 2014;6:504-512. 